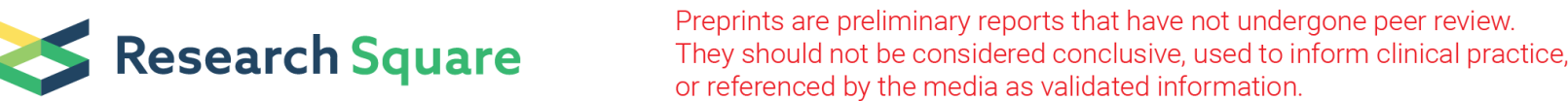

\section{Electron Ultra-High Dose Rate FLASH And Conventional Irradiation Induce Distinct Regulations of Inflammatory Cytokines And CD8 T Lymphocytes Ratio In Mice}

\section{De-Huan Xie}

Sun Yat-sen University Cancer Center

Yi-Chuan Li

Elekta Instrument $A B$

\section{Sai Ma}

Elekta Instrument $A B$

Xin Yang

Sun Yat-sen University Cancer Center

Ruo-Ming Lan

Shandong Normal University School of Physics and Electronics

\section{Yan Mei}

Sun Yat-sen University Cancer Center

\section{Li-Xia Peng}

Sun Yat-sen University Cancer Center

\section{Ao-Qiang Chen}

Sun Yat-sen University Cancer Center

Yan-Hong Lang

Sun Yat-sen University Cancer Center

\section{Zuo-Feng Li}

guangzhou concord cancer center

\section{Bi-Jun Huang}

Sun Yat-sen University Cancer Center

\section{Yan Chen}

Elekta Instrument $A B$

Xiao-Yan Huang

Sun Yat-sen University Cancer Center

Chao-Nan Qian ( $\square$ qianchn@sysucc.org.cn )

Sun Yat-sen University Cancer Center 
Research

Keywords: FLASH irradiation, ultra-high dose rate, cytokine, inflammation

Posted Date: July 27th, 2021

DOI: https://doi.org/10.21203/rs.3.rs-727597/v1

License: (c) (i) This work is licensed under a Creative Commons Attribution 4.0 International License. Read Full License 


\section{Abstract}

Purpose: Ultra-high dose rate FLASH irradiation has been shown to cause less normal tissue damage compared with conventional irradiation, also termed "FLASH effect". However, the underlying mechanism was scarcely known. The purpose of the present study was to determine whether FLASH and conventional irradiation would induce differential inflammatory cytokines expression.

Materials and methods: Female FvB mice were randomly assigned to three different groups: nonirradiated control, conventional (CONV) and FLASH groups. Mice were irradiated at 6 to $19 \mathrm{~Gy}$ of CONV $(0.1 \mathrm{~Gy} / \mathrm{s})$ or FLASH (38.5-600 Gy/s) irradiation using an Elekta Synergy linac $(6 \mathrm{MeV})$. Mice were immobilized in prone position in a custom-designed applicator with dosimetry films positioned under the body. Dose were verified by Gafchromic films. Enzyme linked immunosorbent assay (ELISA) were performed in serum samples of the mice at 6,18 and 31 days after irradiation for four inflammatory cytokines: tumor necrosis factor-a (TNF-a), interferon-y (IFN- - ), interleukin-6 (IL-6) and IL-10. Flow cytometry using antibodies for CD3, CD8, CD4 and CD45 in blood were performed pre-and 1-week post irradiation.

Results: At D6 (18-19 Gy), both IL-6 and TNF-a were elevated, and IL-10 was reduced in FLASH and CONV group, while IFN-y was only significantly increased in conventional group, compared with control group. At D18 (10 Gy) and D31 (13-19 Gy), conventional RT significantly elevated levels of IL-6, IFN- $\gamma$ and TNF-a and reduced IL-10 level compared with FLASH group and control group. Additionally, even low dose conventional irradiation (13 Gy) could induce higher level of pro-inflammatory cytokines and lower level of anti-inflammatory cytokine than high dose (17-19 Gy) FLASH irradiation at D31. Flow cytometry showed that the $\mathrm{CD} 8+/ \mathrm{CD} 45+$ ratio in the blood were higher in the conventional than in FLASH. These data indicate that minor inflammatory cytokine levels of serum in FLASH could be result of the absent of immune overactivation induced by conventional irradiation.

Conclusions: Ultra-high dose rate electron FLASH caused less inflammatory cytokine levels of serum which might be a result from less CD8+/CD45+ ratio in the blood. Thus, differential cytokines and CD8+ T cell expression between FLASH and conventional irradiation would be a potential mechanism for "FLASH effect".

\section{Introduction}

Ultra-high dose rate FLASH radiotherapy (RT) has attracted increased interest because of its potential to spare normal tissue from severe side effects, also termed "FLASH effect"[1-11]. Ultra-high dose rate beams were generated by varying the linac's gun-grid tension, pulse-repetition frequency (PRF), pulse width, and source-to-surface distance (SSD), which have been used to irradiate mice[1-3, 6, 9, 10], minipig, cat-cancer patients[11] and a human CD30 + T-cell cutaneous lymphoma patient[12]. Conventional irradiation increased expression of the dominating pro-inflammatory cytokines IL-6, TNF- $\alpha$ and IL-1 $\beta$, both systemically and in local tissue within radiation range[13-15]. The perpetual inflammatory cytokines 
activation contributes to the generation and development of normal tissue damage such as impaired cognitive function, radiation-induced mucositis and fibrosis $[14,16,17]$.

Despite FLASH-RT presents a promising technology that could improve the delivery of radiotherapy in the future, the limited availability of such devices cannot meet the fast-growing research demands of FLASH related biology studies. Furthermore, the underlying mechanism of "FLASH effect" was scarcely known. The expression of pro-inflammatory and anti-inflammatory cytokines regulated by FLASH and conventional irradiation in animal models was scarcely explored. The purpose of the present study was to determine whether FLASH and conventional irradiation would induce differential inflammatory cytokines expression.

\section{Methods And Materials}

\section{FLASH beam generation}

Our modified linac setup was based on Lempart et al's. [18], with some changes to simplify its implementation. These changes reduced the equipment needs while ensuring a stable dose output. We used the analog signal detection port of the beam generation system reserved for engineers to identify and count the electron beam pulses. Unlike in Lempart's work [18], we, in principle, simplified the signal path of pulse identification to achieve a higher count accuracy by analyzing the picked signals. The test signal could be the magnetron current (MI) or the modulator's pulse forming network (PFN) with a sharp falling edge for each pulse, as seen in Fig. 1a. The test signal passed through a voltage comparator. At a preset number of pulses, the comparator's output was reversed and transmitted to the microcontroller to send a trigger to a high-speed relay, which was connected to the Function Keypad's (FKP) interrupt port. In doing this, we realized the beam termination at a single or any number of pulses as set. To set the delivered pulse number, an 8-bit timer was connected to a microcontroller unit (MCU, AT89C52, Atmel Corporation, San Jose, California, USA) (Fig. 1b), similar to the electrical control unit reported by Lempart et al. [18].

For mice irradiation, the "treatment head" of the linac, or the beam limiting device (BLD), was lifted and removed with the gantry set at 180 degrees. The sample was placed a short distance from the source to increase the dose output and the BLD safety interlocks bypassed. The filament current, magnetic field, and charging current of the magnetron were adjusted to increase the radio frequency's (RF) power. The electron gun's output was fine-tuned while the RF's power was gradually increased so that the starting pulses were stable (Fig. 1a). The energy beam was slightly altered from the clinical $6 \mathrm{MeV}$ beam.

To test if the dose output of each pulse was stable, a repeated single-pulse dose measurement was performed with a PTW Advanced Markus ion chamber (Type 34045, PTW-Freiburg) placed at a distance from the irradiation sample. In order to determine whether the output was stable, a single-pulse beam was repeated 25 times with 10 second intervals. Film measurement was compared with the chamber's measurement for consistency checks. 
Till now, the optimum FLASH parameters are uncertain. Here, we tested two strategies to obtain an ultrahigh dose rate by either a manual repeated single pulse beam with a trigger interval of 20 seconds in between (Fig. 1c upper) or multiple pulses with a 10 millisecond (ms) interval triggered once. The pulse width was 3.3 microsecond ( $\mu \mathrm{s}$ ) and the PRF was $12.5 \mathrm{~Hz}$ for single pulse delivery and $100 \mathrm{~Hz}$ for multiple pulse delivery (Fig. 1c lower). Verification experiments were performed before irradiating the mice.

\section{Film dosimetry}

Gafchromic EBT3 films [Gafchromic ${ }^{\text {TM }}$ EBT3-1417, Lot number 06191801 (expiration date June 2020)] and EBT XD films [Ashland Inc., Bridgewater, NJ 08807, USA, lot number 08221901 (expiration date February 2022)] were used for dose measurement. A study by Jaccard et al. [19] demonstrated response differences to EBT3 film between four electron beams with energies between 6 and $16 \mathrm{MeV}$ was within $0.5 \%$, so we decided to perform optical density (OD) calibration with a $10 \mathrm{MeV}$ electron beam (Elekta Synergy linac) with a $40 \mathrm{~mm} \times 40 \mathrm{~mm}$ field size, $100 \mathrm{~cm} \mathrm{SSD}$, at the maximum dose depth. The dose points for calibration were from 1 to $15 \mathrm{~Gy}$ with $0.5 \mathrm{~Gy}$ increments.

Two calibration curves were generated, one for quick reading to ensure the correct dose was to be delivered to the samples, and one for reading the film scanner (Epson Expression 11000XL, Seiko Epson Corporation, Nagano, Japan) 24-hours post-irradiation. Beam percentage depth dose curve (PDD) and absolute dose at the maximum dose depth of the FLASH beam were measured by two films positioned perpendicularly in the mice applicator (depth $16 \mathrm{~mm}$ ) (Fig. 2a).

Irradiation films were read out with the film scanner 24-hours post-irradiation and analyzed with MEPHYSTO mc $^{2}$ (Medical Physics Tool) (MEPHYSTO mcc 3.3, PTW-Beijing).

\section{Mice irradiation}

This study included 693 to 5 week old female FvB mice (Jackson Laboratory, Sacramento, CA). This study was approved by the Institutional Animal Care and Use Committee of Sun Yat-sen University Cancer Center with the approval number of L102042019080P.

The FVB mice were anesthetized by isoflurane and immobilized in the prone position in a customdesigned applicator, which consisted of lead blocks to position the head, $15 \mathrm{~mm}$ of thick silica gel and a mouse holding plate (Supple. Fig. 1a). Films were placed between the mice and the silica gel to calculate the dose irradiated to the mouse's ventral skin.

For FLASH irradiation, the applicator was placed $2 \mathrm{~cm}$ above the frame of the ionization chamber (SSD $15 \mathrm{~cm}$ ). For conventional irradiation, the SSD was $95 \mathrm{~cm}$ in order to uniformly irradiated two to three mice at a time. The beam first traversed the plastic applicator's $1 \mathrm{~mm}$ thick base and the silica gel build-up before reaching the mice, which ensured that the mice's ventral skin was positioned at the maximum of the depth dose curve (Supple. Fig. 1b). 


\section{Serum inflammatory cytokine quantification}

Blood was collected from the tail-vein or eyeball using EDTA-coated tubes 6, 18 and30 days postirradiation. Cells were removed from plasma by centrifugation for $10 \mathrm{~min}$ at 3000 revolutions per minute $(\mathrm{rpm})$ at $4 \circ \mathrm{C}$. Supernatant was then collected and stored at $-80 \circ \mathrm{C}$. Tumor necrosis factor-a (TNF- $\mathrm{a})$, interferon-y (IFN- $\mathrm{Y}$ ), interleukin-6 (IL-6) and IL-10 was tested using commercial enzyme linked immunosorbent assay (ELISA) kits (Fankew, Shanghai FANKEL Industrial Co., Ltd). All assays were conducted according to the manufacturer's instructions.

\section{Flow cytometry analysis}

Erythrocytes were lysed using a red blood cell lysis buffer (BD Bioscience, 555899). Cell suspensions were filtrated through 70-mm cell strainers (Fisherbrand, 22363548), and then washed and resuspended in a staining buffer (PBS with 2\% FBS). Immune cell expression markers CD3 (Cat\#100204, Biolegend), CD4 (12-0041-82, Biolegend), CD8 (100712, Biolegend) and CD45 (103132, Biolegend) were determined by flow cytometry analyses after surface staining with anti-mouse specific antibodies conjugated with FITC, PE, APC or PerCP-cy5.5. All stained cells were analyzed on a CytoFLEX Flow Cytometer (Beckman Coulter), and the data analyzed using CytExpert software v2.4.

\section{Statistical analysis}

Single dose per pulse, mean dose per pulse, mean dose rate, intra-pulse dose-rate and total delivery time were calculated as follows:

Single dose per pulse $=\frac{\text { Single dose measured by film }}{\text { single pulse number }}$

Mean dose per pulse $=\frac{\text { Total dose measured by film }}{\text { Total pulse number }}$

Mean dose rate $=$ mean dose per pulse $\mathrm{x}$ pulse repetition frequency (mean dose rate for FLASH treatment was modulated by the set PRF)

Intra-pulse dose-rate $=\frac{\text { Mean dose per pulse }}{\text { pulse width (beam-on time })}$

Total delivery time $=$ pulse width $\times$ pulse number + pulse interval time $\times($ pulse number -1$)$

Data were analyzed by using GraphPad Prism. Student's t-test or ANOVA were used to compare the differences between two groups or among more groups. The data were presented as mean \pm standard error. Statistical significance was denoted by $P$ values. Degrees of significance were ${ }^{*} \mathrm{p} \leq 0.05,{ }^{* *} \mathrm{p} \leq$ 0.01 , and ${ }^{\star \star \star} p \leq 0.001$.

\section{Results}




\section{Beam profiles and percentage depth dose curves}

Successive three pulse delivery parameters at a distance from the ion chamber of $0 \mathrm{~cm}, 1.5 \mathrm{~cm}, 3 \mathrm{~cm}$ and $5 \mathrm{~cm}$ were used. Total doses were read out from the films, then the dose per pulse was calculated by the total dose divided by three (Fig. 2b). Successive three, four and five pulse delivery at a distance from the ion chamber of $0 \mathrm{~cm}, 1.5 \mathrm{~cm}, 3 \mathrm{~cm}$ and $5 \mathrm{~cm}$ were used. Total doses were read out from the films (Fig. 2c). For the irradiated area of the mice, the beam's range of flatness was 97\%-105\% (Supple. Figure 2b. blue line), and the maximum dose was at $2 \mathrm{~mm}$ subcutaneous depth (Supple. Figure 3a. red line).

\section{Dose measurement by film}

Forty-four mice were irradiated using three dose levels of 6, 9, 12 and $15 \mathrm{~Gy}$ with a 20 second pulse interval. The mean pulse number was 3.64 (range: $2-5$ pulses). The mean dose per pulse was $3.08 \mathrm{~Gy}$ (range: 2.68-3.48 Gy/pulse), with each pulse delivered with a beam-on time of 3.3 us, resulting in a mean intra-pulse dose-rate of $9.3 \times 10^{5} \mathrm{~Gy} / \mathrm{s}$, while the mean dose rate was $38.5 \mathrm{~Gy} / \mathrm{s}$ (range: $33.5 \sim 43.5 \mathrm{~Gy} / \mathrm{s}$ ) with a PRF of $12.5 \mathrm{~Hz}$. The mean irradiation dose for the $41 \mathrm{film}$ measurements was $11.3 \mathrm{~Gy}$ (range: 5.9$15.4 \mathrm{~Gy}$ ) (irradiation films for three mice were accidentally forgotten). Deviations between the prescribed dose and the film measurement were $2.47 \%$ on average $(-11.93 \% \sim 13.77 \%)$, and $78.0 \%$ of the data $(32 / 41)$ were within $\pm 5 \%$.

For the other 25 mice, using a $10 \mathrm{~ms}$ pulse interval, we were able to deliver a more accurate dose with a mean dose per pulse of $5 \mathrm{~Gy}$ (range: $3.33-6.33 \mathrm{~Gy} /$ pulse), a mean intra-pulse dose rate of $1.52 \cdot 10^{6} \mathrm{~Gy} / \mathrm{s}$ (range: $0.81 \times 10^{6}$ to $1.9 \times 10^{6}$ ) and a mean dose rate of $500 \mathrm{~Gy} / \mathrm{s}$ (range: $30-600 \mathrm{~Gy} / \mathrm{s}$ ) (Table 1). 
Table 1

The two strategy irradiation parameters

\begin{tabular}{|lll|}
\hline Parameters & Strategy 1 & Strategy 2 \\
\hline Number of mice & 44 & 25 \\
\hline Average pulse number & 3.64 pulses (range: $2 \sim 5$ pulses) & constant 3 pulse \\
\hline Average dose per pulse & $\begin{array}{l}3.08 \mathrm{~Gy} \text { (range: } 2.68 \sim 3.48 \\
\text { Gy/pulse) }\end{array}$ & $\begin{array}{l}5 \mathrm{~Gy} \text { (range: } 3.33 \sim 6.33 \\
\text { Gy/pulse) }\end{array}$ \\
\hline Average dose rate & $\begin{array}{l}38.5 \mathrm{~Gy} / \mathrm{s} \text { (range: } 33.5 \sim 43.5 \\
\text { Gy/s) }\end{array}$ & $\begin{array}{l}500 \mathrm{~Gy} / \mathrm{s} \text { (range: } 333 \sim 633 \\
\mathrm{~Gy} / \mathrm{s})\end{array}$ \\
\hline $\begin{array}{l}\text { Average intra-pulse dose- } \\
\text { rate }\end{array}$ & $9.3 \times 10^{5} \mathrm{~Gy} / \mathrm{s}$ & $1.52 \times 10^{6} \mathrm{~Gy} / \mathrm{s}$ \\
\hline Pulse repetition frequency & $12.5 \mathrm{~Hz}$ & $100 \mathrm{~Hz}$ \\
\hline Interval between pulses & Manually $20 \mathrm{~s}$ & $10 \mathrm{~ms}$ \\
\hline Pulse width & Constant 3.3 us & Constant 3.3 us \\
\hline Average total delivery time & $52.8 \mathrm{~s}+12.012$ us & $20 \mathrm{~ms}+9.9$ us \\
\hline
\end{tabular}

\section{Conventional radiotherapy-induced inflammation is reduced by FLASH radiotherapy}

we performed enzyme linked immunosorbent assay (ELISA) in serum samples of the mice for four inflammatory cytokines: IL-6, IFN- $y$, TNF- $a$ and IL-10 6 days post-irradiation (D6, 18-19 Gy), 18 days postIR (D18, $10 \mathrm{~Gy})$ and 31 days post-IR (D31,13-19 Gy).

At D6 (18-19 Gy), both IL-6 and TNF-a were elevated, and IL-10 was reduced in FLASH and CONV group, while IFN- $\gamma$ was only significantly increased in CONV group, compared with non-irradiated group (Fig. 3a). At D18 (10 Gy) and D31 (13-19 Gy), CONV significantly elevated levels of IL-6, IFN-y and TNF-a and reduced IL-10 level compared with FLASH group and non-irradiated group (Fig. 3b-c). Chronologically, IL-6 kept significantly elevated in CONV group, while TNF- $a$ and IL- 6 gradually reduced in FLASH group. However, IL-10 was slightly downregulated at D31 (Fig. 3d-g).

Taken together, FLASH irradiation caused transient elevation of pro-inflammatory cytokines changes and reduction of anti-inflammatory cytokine but gradually recovered to the non-irradiated group level within one month, however, no sign of recovery was seen in the CONV group.

\section{Inflammatory cytokines response reversely in low dose and high dose CONV and FLASH irradiation}


When mice were divided into low dose group (13 Gy) and high dose group (17-19 Gy), different cytokine expression between groups were distinguished. Even low dose CONV irradiation could induce higher level of pro-inflammatory cytokines and lower level of anti-inflammatory cytokine than high dose FLASH irradiation. However, high dose FLASH irradiation induced lower level of anti-inflammatory cytokine compared with non-irradiation group and low dose FLASH irradiation (Fig. 4a-d).

\section{FLASH radiotherapy caused less intense immune response in peripheral blood than conventional radiotherapy}

It has been reported that IFN- $\gamma$ was released from CD8 + T cells [20]. Since IFN- $\gamma$ was only significantly increased in CONV group, we performed flow cytometry to test the CD8+/CD 45 + ratio in three groups. We found that the CD8+/CD45 + ratio in the blood were higher in the CONV-RT than in FLASH-RT (one week post irradiation) (Fig. 5), in accordant with the high level of serum INF-y in CONV group, indicating that more toxicities induced by CONV-RT was as a result of over-activated immune response, which was absent in FLASH-RT.

\section{Discussion}

To our knowledge, this is the first report to compare expression of circulating cytokines in terms of time variation and dose response in ultra-high dose rate electron FLASH- and conventional-irradiated mice. Here, we demonstrated that pro-inflammatory cytokines of IL- 6 and TNF-a were transiently increased and reduced to control level in FLASH, but kept augmenting in conventional group within one month. Another pro-inflammatory cytokine of IFN- $y$ was only significantly increased in CONV group, compared with nonirradiated group and FLASH group. Furthermore, anti-inflammatory cytokine of IL-10 was transitorily decreased in FLASH group while remained downregulated in CONV group. Last but not least, low dose conventional irradiation (13 Gy) could induce higher level of pro-inflammatory cytokines and lower level of anti-inflammatory cytokine than high dose FLASH irradiation (17-19 Gy).

Previous studies suggest that conventional irradiation could trigger a pro-inflammatory response mediated by fluctuant expression of inflammation-regulating cytokines [13-16, 21, 22]. However, only one study reported the neuroprotective of hippocampus with lower pro-inflammatory cytokine levels induced by FLASH than in conventional irradiation 10 weeks post-irradiation [9], similar to the results in the present study. Although both the results point towards decreased inflammation response in FLASHirradiated mice compared to conventional-irradiated mice, the potential mechanism is still not completely clear.

To determine whether immune cells participated in cytokines regulation, we measured CD 8+/CD $45+$ ratio in the blood, and found that CD8+/CD 45 + ratio were higher in the CONV-RT than in FLASH-RT (one week post irradiation). It has been reported that IFN- $\gamma$ was released from CD8 + T cells [20]. In the present study, IFN- $\gamma$ was only significantly increased in conventional group, compared with non-irradiated group and 
FLASH group, indicating the ultra-high dose rate and conventional dose rate could possibly displayed different immunological responses.

In regard of the potential role cytokines play in the pathogenesis of radiation-induced normal tissue injury, the different cytokine response after FLASH and conventional irradiation would conjecturably lead to tissue dysfunction and failure, such as fibrosis, necrosis and vascular injury [22]. Radiation-induced early and late inflammatory responses have been observed in multiple organs and in vitro studies although the underlying regulating mechanisms are not fully understood [13-17, 21-25]. Whether FLASH and conventional irradiation affect radiation-related toxicities differently regulating through inflammatory and immune factors remained to be systematically explored.

From a technical point of view, we successfully generated a FLASH electron beam delivery by increasing the number of pulses through bypassing the linac's ion chambers. Tuning of the linac's operation parameters achieved a dose per pulse (DPP) of $6 \mathrm{~Gy}$ at a certain height above the ion chamber to irradiate mice and cell line samples, which is equivalent to and in some cases higher than some previously reported results $[7,10,11,18,26]$. The intra-pulse dose-rate was around $1 \times 10^{6} \mathrm{~Gy} / \mathrm{s}$, and the mean dose rate was between 33.5-633 Gy/s using a PRF of $12.5 \mathrm{~Hz}$ or $100 \mathrm{~Hz}$ with a $3.3 \mu$ s pulse width. Such high DPP allowed us to generate a higher dose rate in a shorter delivery time. At present, which parameters play a critical role in the "FLASH effect", including mean dose rate, intra-pulse dose rate, total dose, DPP, the interval between two pulses and so on are still under investigated. However, in our study, we modified the Elekta linac to generate similar parameters to most studies, thereby making it suitable for in vivo experiments.

To mimic skin irradiation, we designed a mouse applicator that provided a dose buildup for the targeted ventral skin and used a lead block to protect the head of the mice. We achieved a $97 \%-105 \%$ flatness of the irradiation area (Supple. Figure $2 \mathrm{~b}$. blue line) and a maximum dose of $2 \mathrm{~mm}$ subcutaneous depth (Supple. Figure 3a. red line), which was sufficient for mice ventral skin irradiation. During mice ventral skin irradiation, films for verifying doses were placed under the mice (Supple. Figure 1), thus the film's reading represented the dose to the mice's ventral skin. The average deviation from the prescribed dose determined by the film measurement was $2.47 \%(-11.93 \%-13.77 \%)$, and $78.0 \%(32 / 41)$, measurements were within $\pm 5 \%$ for the first 44 mice using EBT3 films. However, the deviation in the next 25 mice verified by EBT XD films were less than $5 \%$. This might mean that the effective range of reliable dose-response in the EBT XD film is wider than in the EBT3 film, thus more suitable for ultra-high dose verification. Buonanno et al. [27] and Petersson et al. [28] tested different monitors and dosimeters (Gafchromic ${ }^{\text {TM }}$ EBT3, TLD, Alanine pellets, Markus and a custom-made parallel plate ion chamber, and a methyl viologen dosimeter). They concluded that the dose-rate and a level of uncertainty was in the order of $5 \%$ for in-vivo dosimetry. We further compared the immediate dose reading and the reading after 24 hours, and observed that the EBT XD films had more consistent results than the EBT3 films (Fig. 2a).

The current work had certain limitations. Firstly, only four cytokines IL-6, IL-10, IFN-y, and TNF-a was tested. This is partly because IL- 6 and TNF- $a$ were differentially regulated by FLASH and conventional 
irradiation in previous study [9] and partly because they are widely used as principal indicators of the inflammatory response, hence they were suitable to elucidate radiation-related inflammatory changes. Furthermore, the $\mathrm{CD} 8+/ \mathrm{CD} 45$ + ratio in the blood cannot reflect all the distinct impact that the two radiation types have on expression of inflammation-regulating cytokines.

In conclusion, with novel setup and a rigorous laboratory procedure for murine FLASH RT, we verified a distinct circulating pro-inflammatory and anti-inflammatory cytokines were differentially regulated by electron ultra-high dose rate FLASH compared with conventional irradiation chronologically within one month after irradiation. The differential expression of inflammation-regulating cytokines associated with immune response may be the influencing factors of reduced toxicities after FLASH irradiation. Further studies are necessary for elucidating how FLASH and conventional irradiation influence these processes.

\section{Abbreviations}

CONV: conventional

ELISA: Enzyme linked immunosorbent assay

TNF: tumor necrosis factor

IFN: interferon

IL: interleukin

RT: radiotherapy

PRF: pulse-repetition frequency

SSD: source-to-surface distance

MI: magnetron current

PFN: pulse forming network

FKP: Function Keypad

MCU: Microcontroller unit

BLD: beam limiting device

RF: radio frequency

ms: millisecond

$\mu \mathrm{s}$ : microsecond 
OD: optical density

PDD: percentage depth dose curve

rpm: revolutions per minute

\section{Declarations}

\section{Ethical Approval and Consent to participate}

This study was approved by the Institutional Animal Care and Use Committee of Sun Yat-sen University Cancer Center with the approval number of L102042019080P.

Consent for publication

Not applicable.

\section{Availability of supporting data}

None.

\section{Competing interests}

The authors declare that they have no competing interests.

\section{Funding}

This work was supported by grants from the National Natural Science Foundation of China (No. 82073220, No. 81872384 and No. 81672872 to C.Q., No. 81773162 and No. 81572901 to B.H.), the Provincial Natural Science Foundation of Guangdong, China (No.2016A030311011 to C.Q., and No. 2017A030313866 to B.H.), and a research program of Sun Yat-sen University (No. 84000-18843409 to C. Q.).

\section{Authors' contributions}

D.H.X., Y.C., H.X.Y. and C.N.Q. designed the study. D.H.X., Y.C.L, S.M., X.Y., R.M.L., A.Q.C, X.Y.H., and Y. C. modified, manipulated FLASH setting and performed mice irradiation. D.H.X., and Y.M. performed experiments with assistance from A.Q.C., L.X.P. and Y.H.L. D.H.X., Y.C.L., S.M., Z.F.L., B.J.H. and C.N.Q. analyzed and interpreted the data. D.H.X., Y.C.L., S.M., Y.C. and C.N.Q. wrote the manuscript, and revised the manuscript. All authors have reviewed the manuscript and approved the final version.

\section{Acknowledgements}

We thank Elekta Instrument Ltd. (Beijing Branch, Shanghai) for generously providing the linac and other relevant instruments for this study. 


\section{Research and publication ethics}

Not applicable.

\section{Availability of data and material}

The datasets used and/or analyzed in this study were uploaded onto the Research Data Deposit public platform (www.researchdata.org.cn) with the RDD approval number RDDB2020001032.

\section{References}

1. Alaghband Y, Cheeks SN, Allen BD, et al. Neuroprotection of Radiosensitive Juvenile Mice by UltraHigh Dose Rate FLASH Irradiation. Cancers (Basel). 2020;12(6):1671. Published 2020 Jun 24. doi:10.3390/cancers12061671.

2. Allen BD, Acharya MM, Montay-Gruel P, et al. Maintenance of Tight Junction Integrity in the Absence of Vascular Dilation in the Brain of Mice Exposed to Ultra-High-Dose-Rate FLASH Irradiation. Radiat Res. 2020;194(6):625-35. doi:10.1667/RADE-20-00060.1.

3. Favaudon V, Caplier L, Monceau V, et al. Ultrahigh dose-rate FLASH irradiation increases the differential response between normal and tumor tissue in mice [published correction appears in Sci Transl Med. 2019 Dec 18;11(523):]. Sci Trans/ Med. 2014;6(245):245ra93. doi:10.1126/scitranslmed.3008973.

4. Fouillade C, Curras-Alonso S, Giuranno L, et al. FLASH Irradiation Spares Lung Progenitor Cells and Limits the Incidence of Radio-induced Senescence. Clin Cancer Res. 2020;26(6):1497-506. doi:10.1158/1078-0432.CCR-19-1440.

5. Kim YE, Gwak SH, Hong BJ, et al. Effects of Ultra-high dose rate FLASH Irradiation on the Tumor Microenvironment in Lewis Lung Carcinoma: Role of Myosin Light Chain. Int J Radiat Oncol Biol Phys. 2021;109(5):1440-53. doi:10.1016/j.ijrobp.2020.11.012.

6. Levy K, Natarajan S, Wang J, et al. Abdominal FLASH irradiation reduces radiation-induced gastrointestinal toxicity for the treatment of ovarian cancer in mice. Sci Rep. 2020;10(1):21600. doi:10.1038/s41598-020-78017-7. Published 2020 Dec 10.

7. Montay-Gruel P, Acharya MM, Petersson K, et al. Long-term neurocognitive benefits of FLASH radiotherapy driven by reduced reactive oxygen species [published correction appears in Proc Natl Acad Sci U S A. 2020 Oct 13;117(41):25946-25947]. Proc Natl Acad Sci U S A. 2019;116(22):1094310951. doi:10.1073/pnas.1901777116.

8. Montay-Gruel P, Petersson K, Jaccard M, et al. Irradiation in a flash: Unique sparing of memory in mice after whole brain irradiation with dose rates above 100Gy/s. Radiother Oncol. 2017;124(3):365-9. doi:10.1016/j.radonc.2017.05.003.

9. Simmons DA, Lartey FM, Schüler E, et al. Reduced cognitive deficits after FLASH irradiation of whole mouse brain are associated with less hippocampal dendritic spine loss and neuroinflammation. Radiother Oncol. 2019;139:4-10. doi:10.1016/j.radonc.2019.06.006. 
10. Soto LA, Casey KM, Wang J, et al. FLASH Irradiation Results in Reduced Severe Skin Toxicity Compared to Conventional-Dose-Rate Irradiation. Radiat Res. 2020;194(6):618-24. doi:10.1667/RADE-20-00090.

11. Vozenin MC, De Fornel P, Petersson K, et al. The Advantage of FLASH Radiotherapy Confirmed in Mini-pig and Cat-cancer Patients. Clin Cancer Res. 2019;25(1):35-42. doi:10.1158/1078-0432.CCR17-3375.

12. Bourhis J, Sozzi WJ, Jorge PG, et al. Treatment of a first patient with FLASH-radiotherapy. Radiother Oncol. 2019;139:18-22. doi:10.1016/j.radonc.2019.06.019.

13. Nielsen S, Bassler N, Grzanka L, et al. Proton scanning and X-ray beam irradiation induce distinct regulation of inflammatory cytokines in a preclinical mouse model. Int $\mathrm{J}$ Radiat Biol. 2020;96(10):1238-44. doi:10.1080/09553002.2020.1807644.

14. Ong ZY, Gibson RJ, Bowen JM, et al. Pro-inflammatory cytokines play a key role in the development of radiotherapy-induced gastrointestinal mucositis. Radiat Oncol. 2010;5:22. doi:10.1186/1748717X-5-22. Published 2010 Mar 16.

15. Rübe $C E$, Wilfert $F$, Palm J, et al. Irradiation induces a biphasic expression of pro-inflammatory cytokines in the lung. Strahlenther Onkol. 2004;180(7):442-8. doi:10.1007/s00066-004-1265-7.

16. Dong X, Luo M, Huang G, et al. Relationship between irradiation-induced neuro-inflammatory environments and impaired cognitive function in the developing brain of mice. Int $\mathrm{J}$ Radiat Biol. 2015;91(3):224-39. doi:10.3109/09553002.2014.988895.

17. Rübe $C E$, Uthe $D$, Wilfert $F$, et al. The bronchiolar epithelium as a prominent source of proinflammatory cytokines after lung irradiation. Int J Radiat Oncol Biol Phys. 2005;61(5):1482-92. doi:10.1016/j.jjrobp.2004.12.072.

18. Lempart M, Blad B, Adrian G, et al. Modifying a clinical linear accelerator for delivery of ultra-high dose rate irradiation. Radiother Oncol. 2019;139:40-5. doi:10.1016/j.radonc.2019.01.031.

19. Jaccard M, Petersson K, Buchillier T, et al. High dose-per-pulse electron beam dosimetry: Usability and dose-rate independence of EBT3 Gafchromic films. Med Phys. 2017;44(2):725-35. doi:10.1002/mp.12066.

20. Wang W, Green M, Choi JE, et al. CD8 + T cells regulate tumour ferroptosis during cancer immunotherapy. Nature. 2019;569(7755):270-4. doi:10.1038/s41586-019-1170-y.

21. Nielsen S, Bassler N, Grzanka L, et al. Comparison of Coding Transcriptomes in Fibroblasts Irradiated With Low and High LET Proton Beams and Cobalt-60 Photons. Int J Radiat Oncol Biol Phys. 2019;103(5):1203-11. doi:10.1016/j.ijrobp.2018.11.065.

22. Zhao W, Robbins ME. Inflammation and chronic oxidative stress in radiation-induced late normal tissue injury: therapeutic implications. Curr Med Chem. 2009;16(2):130-43. doi:10.2174/092986709787002790.

23. Rübe $C E$, Wilfert $F$, Uthe $D$, et al. Increased expression of pro-inflammatory cytokines as a cause of lung toxicity after combined treatment with gemcitabine and thoracic irradiation. Radiother Oncol. 2004;72(2):231-41. doi:10.1016/j.radonc.2004.05.004. 
24. Wang XS, Shi Q, Williams LA, et al. Inflammatory cytokines are associated with the development of symptom burden in patients with NSCLC undergoing concurrent chemoradiation therapy. Brain Behav Immun. 2010;24(6):968-74. doi:10.1016/j.bbi.2010.03.009.

25. Cho HJ, Lee WH, Hwang OMH, Sonntag WE, Lee YW. Role of NADPH oxidase in radiation-induced pro-oxidative and pro-inflammatory pathways in mouse brain. Int J Radiat Biol. 2017;93(11):125766. doi:10.1080/09553002.2017.1377360.

26. Patriarca A, Fouillade C, Auger M, et al. Experimental Set-up for FLASH Proton Irradiation of Small Animals Using a Clinical System. Int J Radiat Oncol Biol Phys. 2018;102(3):619-26. doi:10.1016/j.jirobp.2018.06.403.

27. Buonanno M, Grilj V, Brenner DJ. Biological effects in normal cells exposed to FLASH dose rate protons. Radiother Oncol. 2019;139:51-5. doi:10.1016/j.radonc.2019.02.009.

28. Petersson K, Jaccard M, Germond JF, et al. High dose-per-pulse electron beam dosimetry - A model to correct for the ion recombination in the Advanced Markus ionization chamber. Med Phys. 2017;44(3):1157-67. doi:10.1002/mp.12111.

\section{Figures}




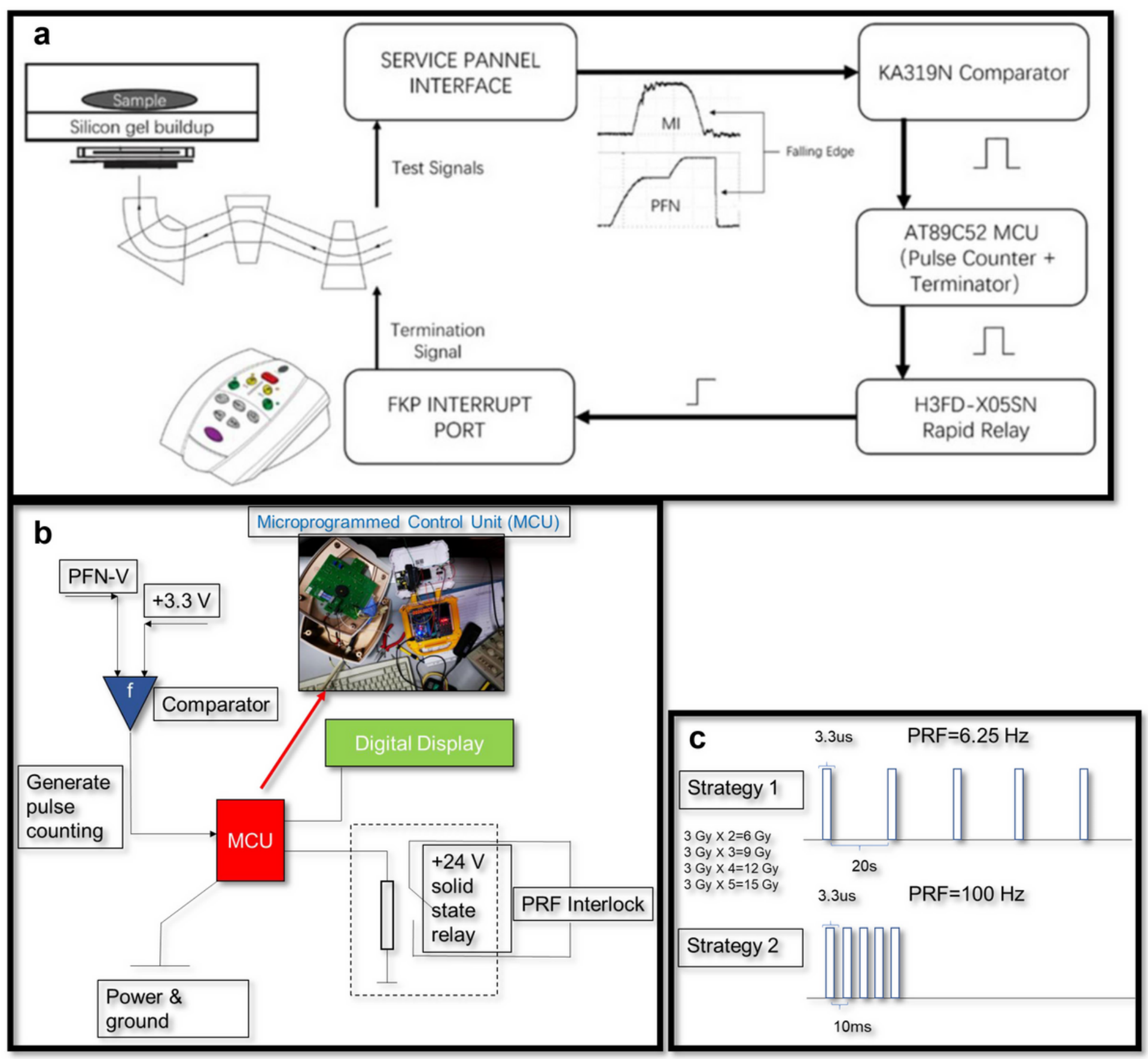

Figure 1

Linac accelerator and mice applicator setup. (a) Schematic diagram of the FLASH proton radiation therapy setup. (b) Schematic diagram of the microprogrammed control unit (MCU). (c) Electrical control device and pulsed FLASH-RT delivery strategy. Strategy 1: pulse repetition frequency (PRF), pulse width and pulse interval time were $6.25 \mathrm{~Hz}, 3.3$ us and 20s. Strategy 2: PRF, pulse width and pulse interval time were $100 \mathrm{~Hz}, 3.3$ us and $10 \mathrm{~ms}$. 


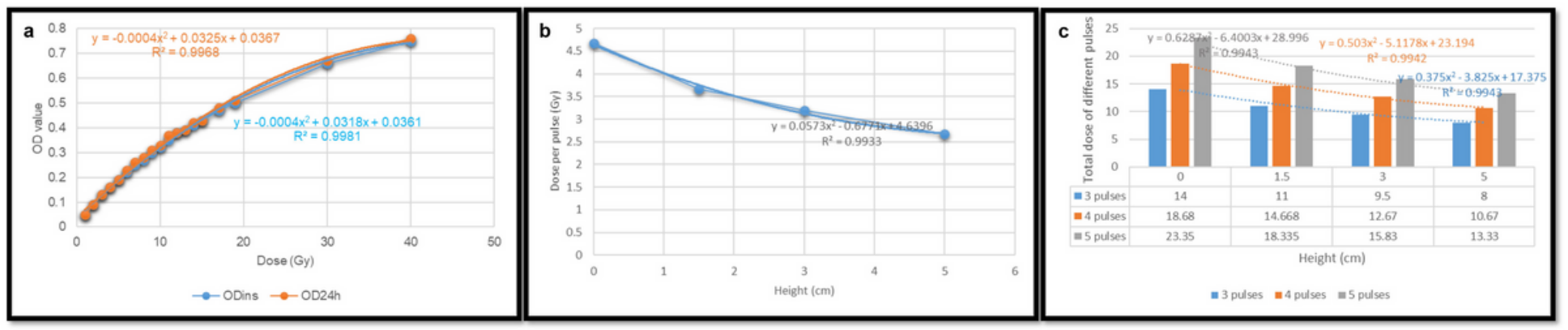

\section{Figure 2}

EBT-XD film calibration, measurements of dose per pulse and total dose of different pulses for different heights from the ion chamber (a) EBT-XD Gafchromic films were used to generate a calibration curve calibrated by $10 \mathrm{MeV}$ electron beam curve. The films were scanned instantly (blue line) and 24 hours (orange line) after exposure with a densitometer and Epson 11000XL scanner, respectively. (b) Successive three pulse delivery at a distance from the ion chamber of $0 \mathrm{~cm}, 1.5 \mathrm{~cm}, 3 \mathrm{~cm}$ and $5 \mathrm{~cm}$. Total doses were read out from the films, then the dose per pulse calculated by the total dose divided by three. (c) Successive three, four and five pulse delivery with a distance from the ion chamber of $0 \mathrm{~cm}, 1.5 \mathrm{~cm}, 3$ $\mathrm{cm}$ and $5 \mathrm{~cm}$. Total doses were read out from the films.

a

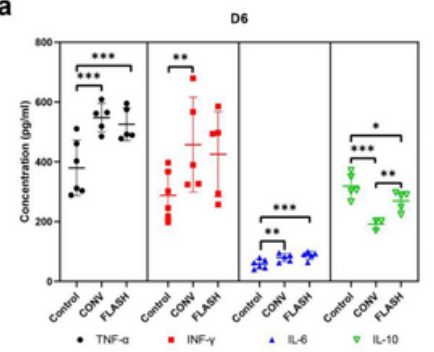

c

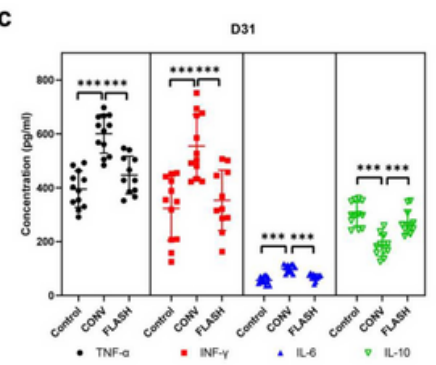

b

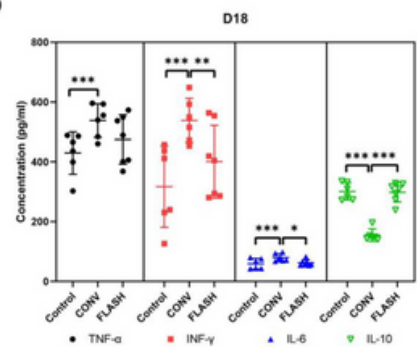

d

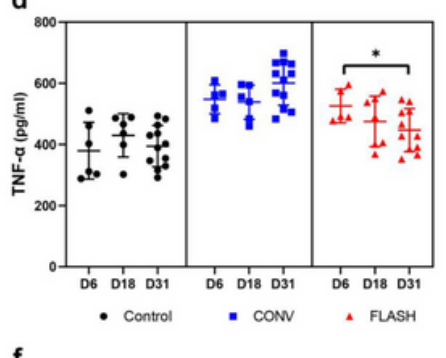

f

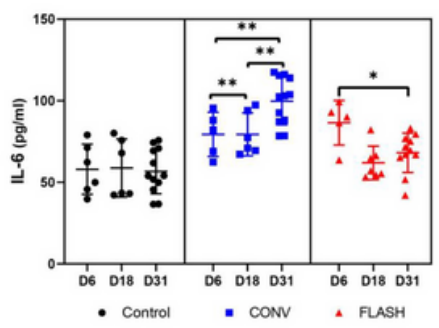

e

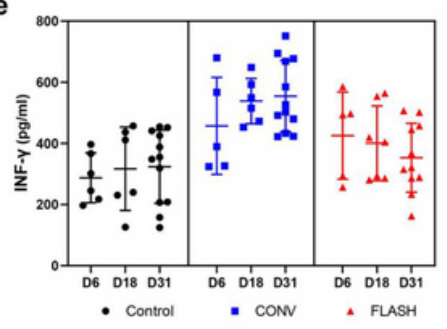

g

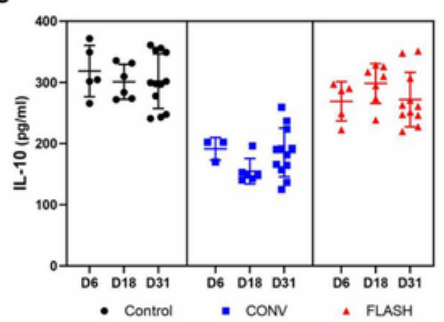

Figure 3

Conventional radiotherapy-induced inflammation is reduced by FLASH radiotherapy (a-c) At D6 (18-19 Gy), both IL- 6 and TNF-a were elevated, and IL-10 was reduced in FLASH and CONV group, while IFN- $y$ was only significantly increased in CONV group, compared with non-irradiated group. At D18 (10 Gy) and D31 (13-19 Gy), CONV significantly elevated levels of IL-6, IFN-y and TNF-a and reduced IL-10 level compared with FLASH group and non-irradiated group. (d-g) In CONV group, IL-6 kept significantly elevated in CONV group. TNF-a and IL-6 gradually reduced in FLASH group. However, IL-10 was slightly downregulated at D31. Results are plotted as mean \pm SEM. Statistical significance of differences 
between treatment groups was determined using a one-way ANOVA with Fisher's LSD post-hoc test. *P < $0.05, * * P<0.01$ and $* * * P<0.001$.

a

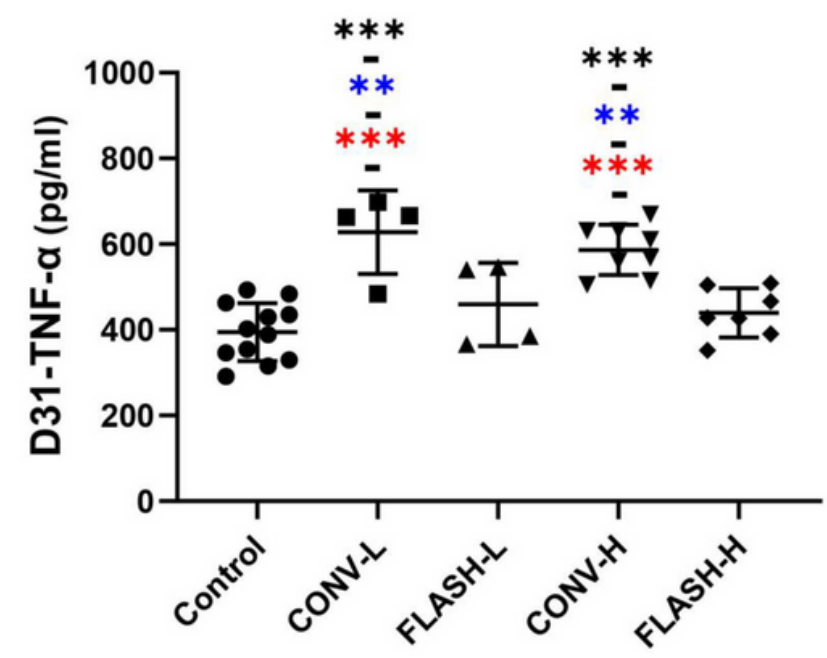

C

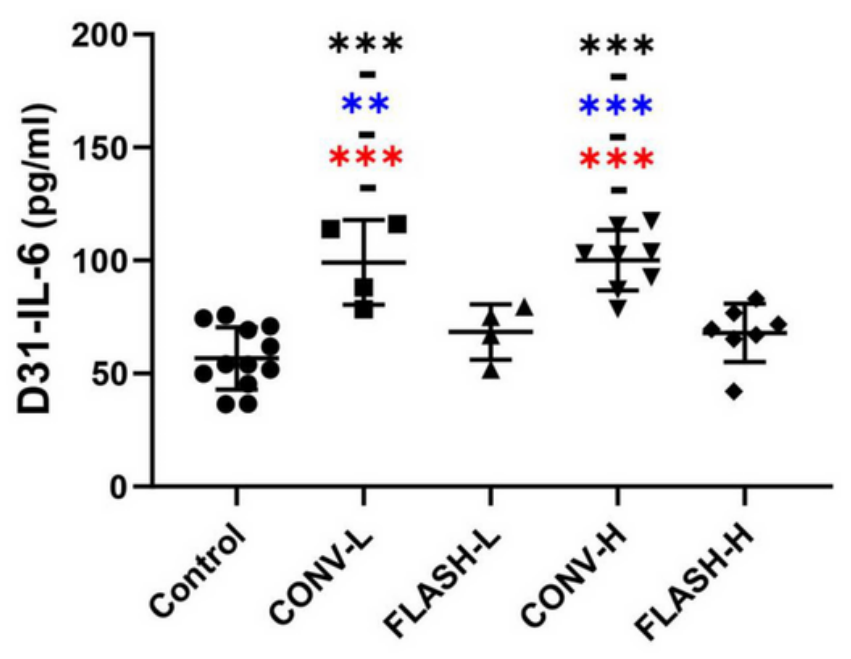

b

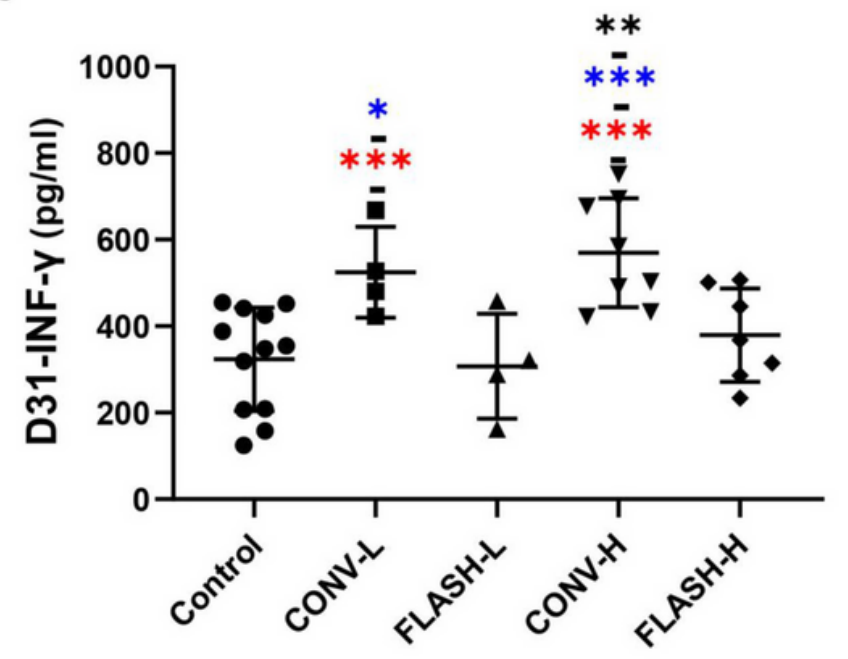

d

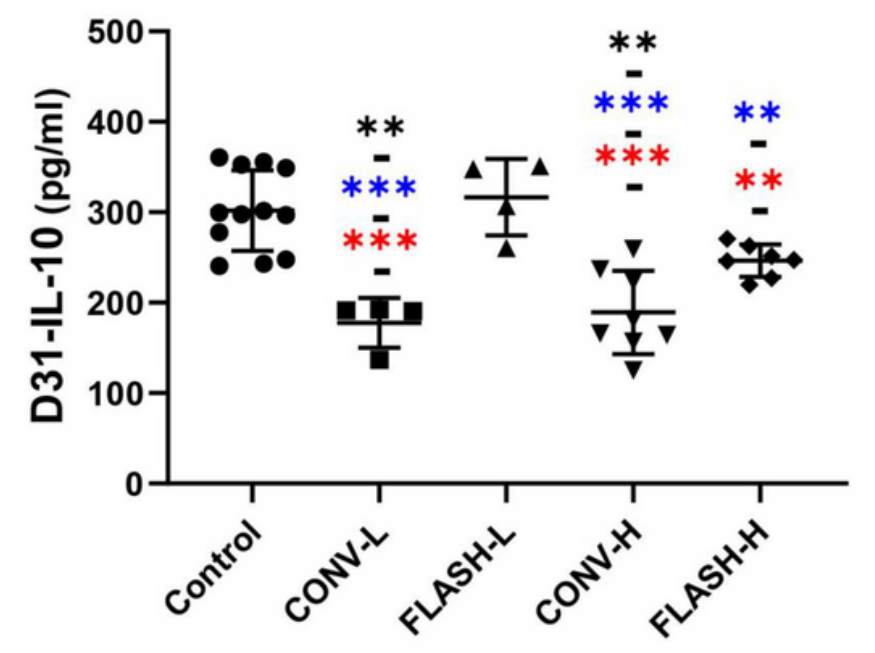

Figure 4

Inflammatory cytokines response reversely in low dose and high dose CONV and FLASH irradiation (a-c) At D31, low dose CONV irradiation significantly induced higher level of pro-inflammatory cytokines (TNF-a and IL-6) and lower level of anti-inflammatory cytokine (IL-10) than high dose FLASH irradiation. However, high dose FLASH irradiation induced lower level of IL-10 compared with non-irradiation group and low dose FLASH irradiation (d). Results are plotted as mean \pm SEM. Statistical significance of differences between treatment groups was determined using a one-way ANOVA with Fisher's LSD posthoc test. ${ }^{*} \mathrm{P}<0.05,{ }^{\star} \mathrm{P}<0.01$ and ${ }^{\star \star *} \mathrm{P}<0.001$. Note: $\mathrm{L}$, low dose (13 Gy); $\mathrm{H}$, high dose (17-19 Gy); red stars, compared with control; bule stars, compared with FLASH-L; black stars, compared with FLASH-H. 

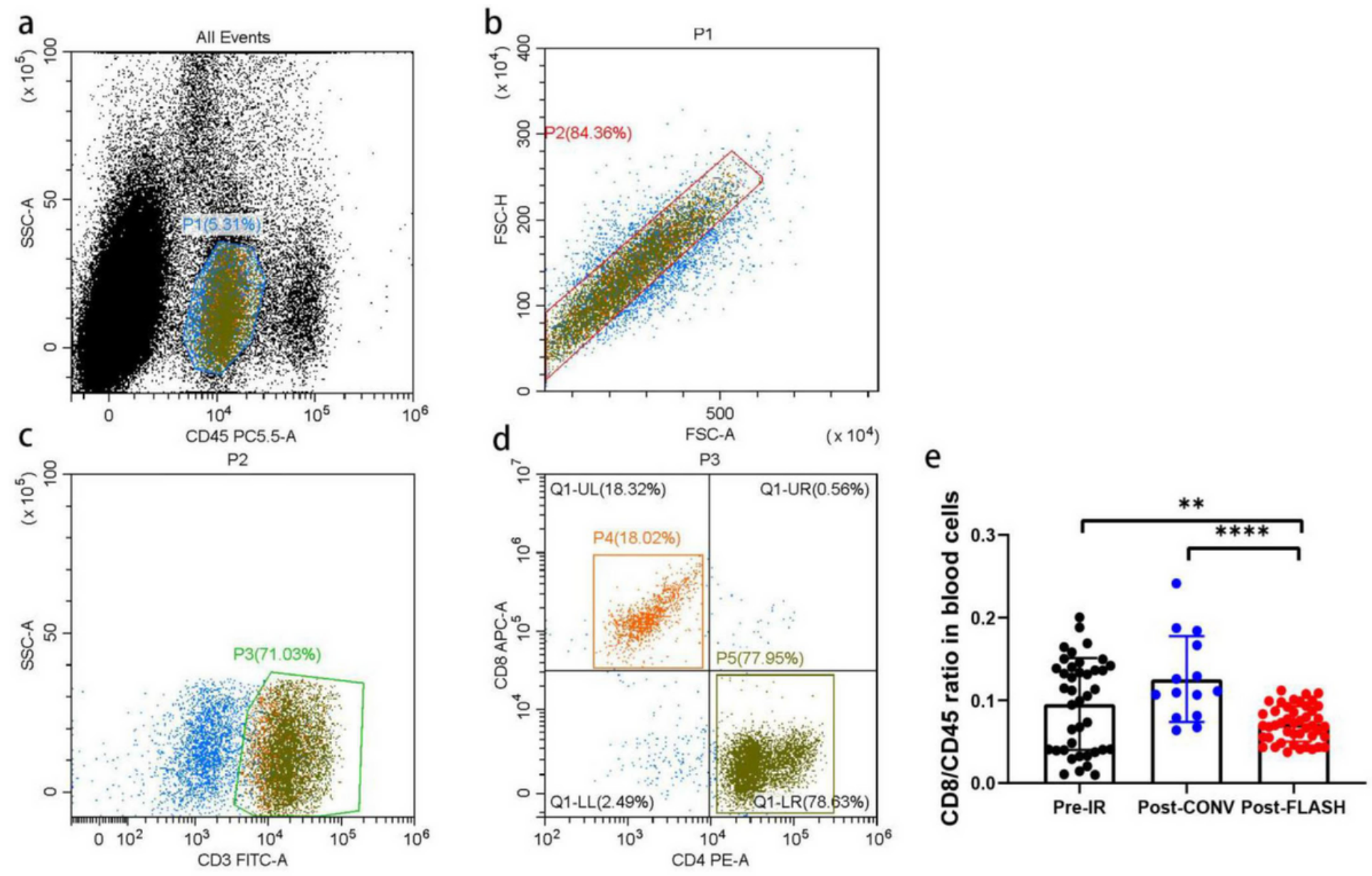

Figure 5

FLASH radiotherapy caused less intense immune response in peripheral blood than conventional radiotherapy (a-d) Flow cytometry analysis of CD45+ cells and CD8+ T cells isolated from peripheral blood. (e) one week post irradiation, CD8+/CD45+ ratio was higher in the CONV-RT than in FLASH-RT.

\section{Supplementary Files}

This is a list of supplementary files associated with this preprint. Click to download.

- Supple.Fig.1.jpg

- Supple.Fig.2.jpg

- Supple.Fig.3.jpg 